www.jmscr.igmpublication.org

Index Copernicus Value: 79.54

ISSN (e)-2347-176x ISSN (p) 2455-0450

crossrefDOI: https://dx.doi.org/10.18535/jmscr/v7i3.163

\title{
A Hospital Based Study of Ponseti Method versus Accelerated Ponseti Method as Conservative Treatment for Congenital Talipes Eqinovarus
}

\author{
Authors \\ Suraj Kumar Jain, Abhishek Sahu \\ V.I.M.S.A.R., Burla
}

\begin{abstract}
Objective: To compare between Ponseti method and accelerated Ponseti method as conservative treatment of ctev by assessing Pirani scoring system in terms of recovery, time taken, number of serial castings and requirement of tenotomy.

Method: Prospective Randomized controlled trial where 80 patients randomly selected into 2 groups 40 each, Group A treated by Ponseti method and Group B treated by accelerated Ponseti method. Group A underwent weekly manipulation and cast and Group $B$ underwent twice weekly manipulation and cast. Pirani score was documented at every visit at presentation, before serial casting after removal of previous cast till Pirani score less than 1. Then after followed by tenotomy and final cast.

Results: $40 \%$ cases were bilateral.63\% male child were affected. All deformities were corrected in $92 \%$ in Group A and $90 \%$ in Group B. Average no. of casts required were 5.5 in Group A and 5.8 in Group B. The difference was found to statistically not significant.

Conclusion: Accelerated ponseti method is as good conservative method for ctev with added advantages of shorter duration of casting and speedy recovery.

Keywords: Accelerated Ponseti, clubfoot, standard Ponseti.
\end{abstract}

\section{Introduction}

Congenital talipes equinovarus (CTEV) occurs in 1.2 per 1000 live births. ${ }^{1}$ In approximately $20 \%$ of patients, CTEV is associated with other congenital abnormalities. $^{2,3}$ A variety of classification systems based on clinical examination have been used, the most widely used being that of Pirani, which has been shown to have good inter observer reliability and reproducibility. ${ }^{4,5}$ The Ponseti method has transformed the management of children with CTEV producing good long-term results and has gained world- wide acceptance ${ }^{6,7}$ The standard Ponseti method uses weekly foot and leg plaster changes to gradually correct the deformity, using a strictly defined sequence of moulded plaster changes. The final deformity to be corrected is equinus, which often requires a percutaneous Tendo-Achillis tenotomy followed by a final plaster for three weeks. Once plastering is finished, children are placed in a foot-abduction brace. Percutaneous Tendo-Achilles tenotomy and later tibialis anterior transfer may be used but are not always necessary.

The Accelerated Ponseti method uses twice weekly casts is as effective as standard Ponseti method and this could reduce hospital stay.

This study was done to compare between Ponseti method and accelerated Ponseti method as 
conservative treatment of CTEV with respect to assessing Pirani scoring system in terms of recovery, time taken for deformity correction, no. of serial casting done andrequirement of tenotomy hence determining the effectiveness of twice weekly casting.

\section{Materials and Methods}

A prospective randomised controlled trial was drawn up in our institute to compare an accelerated Ponseti method with the standard Ponseti method. Ethical approval was obtained from the VIMSAR Ethics Committee, Burla. 80 patients were selected as per inclusion criteria. Inclusion criteria were a diagnosis of idiopathic CTEV, age $<90$ days, and informed consent from the patient's guardian or parents.

A thorough general examination was carried out in order to identify any associated congenital abnormality.

Randomisation occurred at the patient level as those patients whose parents couldn't afford transportation fares twice per week or very poor patients were excluded. All feet were scored using the Pirani method. All 80 patients were divided into 2 groups manipulated either by standard Ponseti method or by Accelarated Ponseti method of 40 each.

Plaster treatment was continued until clinical correction was achieved. A percutaneous Tendo Achillis tenotomy was performed if dorsiflexion was $<10^{\circ}$ at the end of manipulation and plastering. Both groups were put into plaster, following tenotomy, for three weeks. Both groups were given abduction braces to wear for three years following their plaster treatment, 23 hours per day for the first three months, followed by night-time bracing until the child's third birthday. Data analysis was carried out using STATA version 10.0. In order to compare randomised groups for categorical data we used chi-squared tests and Mann-Whitney $U$ tests for continuous variables. A p-value $<0.05$ was considered statistically significant.

\section{Results}

80 children with ctev were taken for study either by standard or accelerated Ponseti method. $40 \%$ cases were bilateral. $63 \%$ male child were affected.

Both the groups were compared and there were no significant differences between the groups before treatment and no significant difference in the final Pirani score. There was no difference between the number of plaster changes between the groups: median 5.8(four to eight) for the accelerated group and 5.4 (four to seven) for the control group.

In the accelerated group 4 of the 40 patients did not respond to the treatment and crossed over to the standard Ponseti method. Two of them required tibialis anterior transfer, whereas the other two achieved correction with weekly manipulation and plaster changes.

In the standard group 3 of 40 patients did not respond to treatment and needed tibialis anterior transfer. 


\begin{tabular}{|l|c|c|c|}
\hline & $\begin{array}{l}\text { Accelerated group } \\
(\mathbf{n = 4 0})\end{array}$ & $\begin{array}{l}\text { Standard group } \\
(\mathbf{n = 4 0})\end{array}$ & P values \\
\hline Median age (in days) & 29 & 34 & 0.34 \\
\hline Number of male(\%) & $26(65)$ & $24(60)$ & 0.78 \\
\hline Bilateral (\%) & $17(42.5)$ & $15(37.5)$ & 0.89 \\
\hline Tenotomy & 33 & 27 & 0.09 \\
\hline Median Pirani score at start & 5.2 & 5 & 0.10 \\
\hline Median Pirani score at tenotomy & 0.75 & 0.5 & 0.3 \\
\hline $\begin{array}{l}\text { Median Pirani score at 6 months } \\
\text { follow up }\end{array}$ & 0.3 & 0.5 & 0.7 \\
\hline
\end{tabular}
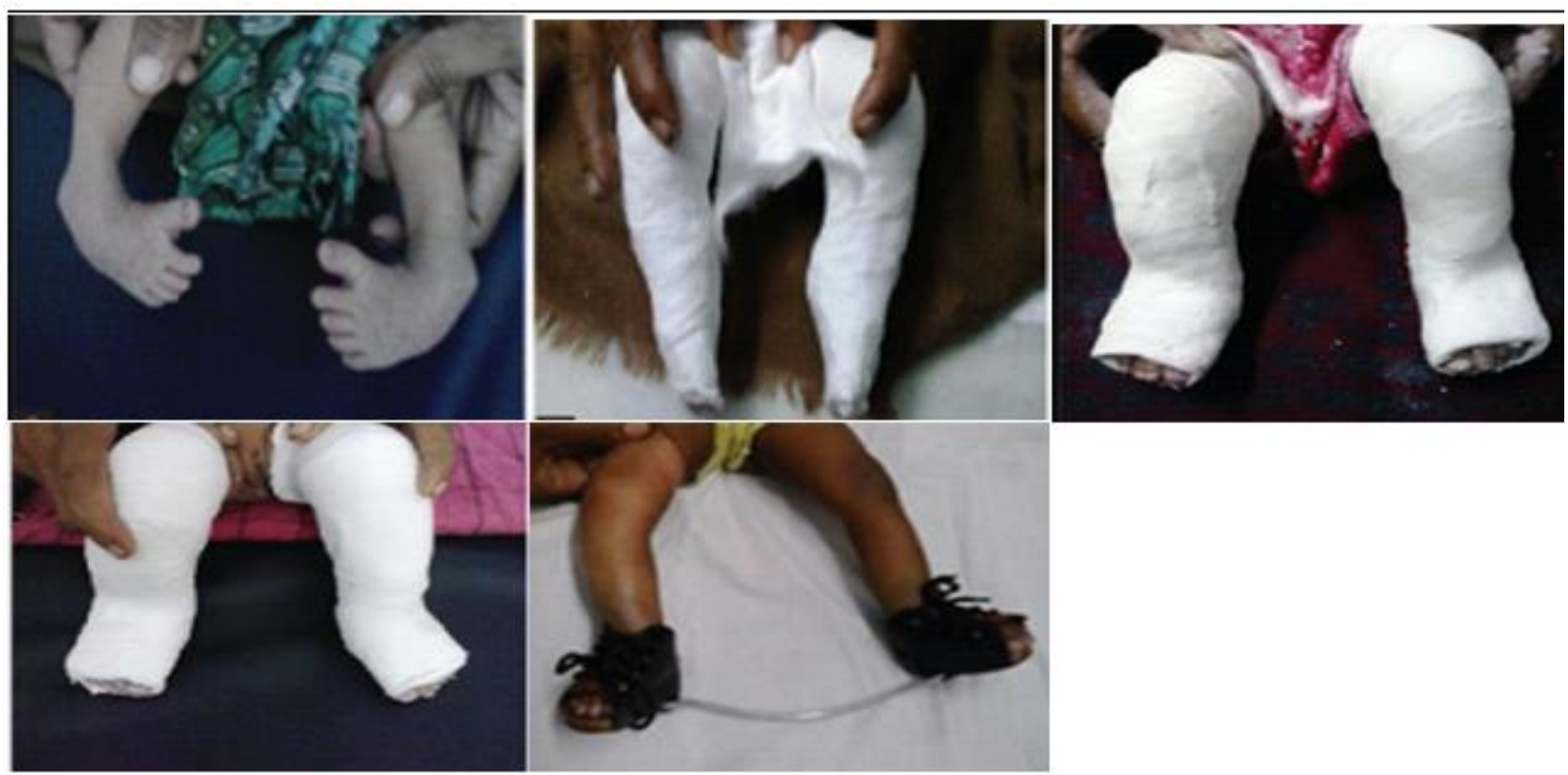

Sequence of correction of CTEV in a 30 day infant (from top-right to bottom-left)

\section{Discussion}

The Ponseti method is widely accepted and practised, giving reliably good long-term results. Our results suggest that in the short term comparable results can be achieved with an accelerated method, changing the plaster two times per week. The tenotomy rate in the accelerated group was $80 \%$, compared to $65 \%$ in the control group $(\mathrm{p}=0.08)$, which was not statistically significant. A number of authors have described a percutaneous tendo Achillis tenotomy rate for the correction of persistent equinus using the Ponseti method of $70 \%$ to $80 \%$.
In our study both study groups had similar range of deformity $(\mathrm{p}=0.1)$.

Tibialis anterior transfer was required in 4 of the 80 patients $(5 \%)$ to achieve correction.

Morcuende et al. reviewed more than 230 patients and concluded that the results of 5 days casting and 7 days casting were same. ${ }^{7}$

Harnett et al. reviewed thrice weekly and once weekly casting and concluded that both were equally effective. They took into consideration 40 patients and found that Pirani score decreased by an average of 4.5 in accelerated group and 4 in conventional group. ${ }^{8}$ 
The limitation of our study were we took into consideration less than 90 days old babies and patients without syndromic CTEV.

\section{Conclusion}

The accelerated Ponseti method with plaster changes two times a week appears to be as effective as the standard Ponseti method in ctev with added advantages of shorter duration of casting and speedy recovery.

\section{References}

1. Mkandawire NC, Kaunda E. Incidence and patterns of congenital talipes equinovarus (clubfoot) deformity at Queen Elizabeth Central Hospital, Banter, Malawi. East Afr J Surg2004;9:28-31.

2. Siapkara A, Duncan R. Congenital talipes equinovarus: a review of current management. J Bone Joint Surg [Br] 2007;89-B:995-1000.

3. Chung CS, Nemechek RW, Larsen IJ, Ching GH. Genetic and epidemiological studies of clubfoot in Hawaii: general and medical considerations. Hum Hered1969;19:321-42.

4. Pirani S. Pirani severity scoring. In: Staheli I, ed. Clubfoot: Ponseti management. Third ed. Global-HELP, 2009:27. http://www.global-help.org/ Publications/books/ (date last accessed 22 November 2010).

5. Flynn JM, Donohue M, Mackenzie WG. An independent assessment of two clubfoot-classification systems. J PediatrOrthop1998;18:323-7.

6. Cooper DM, Dietz FR. Treatment of idiopathic clubfoot: a thirty-year follow-up note. J Bone Joint Surg [Am] 1995;77A:1477-89.
7. Morcuende JA, Dolan LA, Dietz FR, Ponseti IV. Radical reduction in the rate of extensive corrective surgery for clubfoot using the Ponseti method. Pediatrics 2004;113:376-80.

8. Harnett $\mathrm{P}$, Freeman R, Harrison WJ, Brown LC, Beckles V. An accelerated Ponseti versus the standard Ponseti method: A prospective randomised controlled trial. J Bone Joint Surg $\mathrm{Br}$ 2011;93:404-8. 